\title{
Optimization of heterodyne observations using Allan variance measurements
}

\author{
R. Schieder and C. Kramer \\ I. Physikalisches Institut, Universität zu Köln, Zülpicher Straße 77, 50937 Köln, Germany \\ Received 11 January 2001 / Accepted 26 April 2001

\begin{abstract}
Stability tests based on the Allan variance method have become a standard procedure for the evaluation of the quality of radio-astronomical instrumentation. They are very simple and simulate the situation when detecting weak signals buried in large noise fluctuations. For the special conditions during observations an outline of the basic properties of the Allan variance is given, and some guidelines how to interpret the results of the measurements are presented. Based on a rather simple mathematical treatment clear rules for observations in "Position-Switch", "Beam-" or "Frequency-Switch", "On-The-Fly-" and "Raster-Mapping" mode are derived. Also, a simple "rule of the thumb" for an estimate of the optimum timing for the observations is found. The analysis leads to a conclusive strategy how to plan radio-astronomical observations. Particularly for air- and space-borne observatories it is very important to determine, how the extremely precious observing time can be used with maximum efficiency. The analysis should help to increase the scientific yield in such cases significantly.
\end{abstract}

Key words. instrumentation: miscellaneous - methods: data analysis, observational - space vehicles: instruments - techniques: spectroscopic - telescopes

\section{Introduction}

Allan variance measurements have been demonstrated as a useful tool for the characterization of the stability of radio-astronomical equipment such as Millimeter or Submillimeter-receivers or large bandwidth back-ends (Schieder et al. 1985; Kooi et al. 2000). Particularly for the development of acousto-optical spectrometers (AOS) at the Kölner Observatorium für Sub-Millimeter Astronomy (KOSMA) the method has played a very important role, because it provides clear evidence that the spectrometers are well suited for the use at an observatory by means of a reliable test laboratory procedure (Tolls et al. 1989). The simple definition of the Allan variance makes it very easy to apply such measurements also for the characterization of the stability of other instruments, a very elementary case is the definition of the quality of a simple Lock-In amplifier for example.

For a real time spectrometer, as used in radioastronomy with many simultaneously operating frequency channels, it is a very important condition that all channels are behaving identically in a statistical sense. Therefore, the use of the Allan variance for the investigation of the performance of the spectrometer is based on the assumption that there are no differences between different frequency channels. That this is not always correct is evi-

Send offprint requests to: R. Schieder,

e-mail: schieder@ph1.uni-koeln.de dent. Thus, it is always necessary to verify the similarity of all frequency channels of the spectrometer by investigating the baseline noise of measured spectra for example. Typical problem areas for instance are light scatter problems in acousto-optical spectrometers (AOS), where speckles may affect individual channels more heavily than others. The same is true for filterbanks which have occasionally same peculiar channels even in a well maintained back-end system. But in all normal cases of well behaved instrumentation, the Allan variance plot is a most useful method to precisely characterize the instrumentation in use.

In general, observations at an observatory are done with the available instrumentation as is, and it can not be modified or even improved by the observer. On the contrary, the observer has to find the correct observing parameters in order to use the available hardware in a most economic way. It is the purpose of this paper to develop a strategy for an optimization of the observing process. For this the knowledge of the stability parameters is decisive. Once this information is available from an Allan variance measurement for example, it should be a rather straightforward matter to determine the essential parameters like length of integration per position on sky et cetera. The following mathematical treatment analyses the commonly used observing methods, i.e. "Position-", "Beam-" or "Frequency-Switch", "On-The-Fly" (OTF) measurements or "Raster-Mapping" based on the 
information contained in the Allan variance plot. As a result practical guidelines for the most efficient observing method are found, which can be used at any radio observatory. Particularly, all space- or air-borne observatories require a most efficient use of the extremely precious observing time, since any loss can usually not be compensated by a simple increase in observatory time. But also for ground-based observatories the results found in the following should be very useful.

\section{Definition of the Allan variance}

If a test procedure is defined for use at any time and at any location, it needs to be as simple and unique as possible. Therefore, we understand the Allan variance as the ordinary statistical variance of the difference of two contiguous measurements (see also Rau \& Schieder 1984). One has to consider a signal-function $s(t)$, which is the instantaneous output signal of a spectrometer channel or of a continuum detector for example. The output is now integrated for a time interval $T$ representing an estimate of the mean signal which is stored as spectrometer data in the computer:

$x(T, t)=1 / T \int_{t-T}^{t} s\left(t^{\prime}\right) \mathrm{d} t^{\prime}$.

The expectation value of $x(T, t)$ is therefore identical with the expectation of $s(t)$. For the observation of weak signals, a certain number $N$ of differences of two of these data, a "signal-measurement" $x_{\mathrm{s}}$ and a "referencemeasurement" $x_{\mathrm{r}}$, are subtracted from each other:

$d=x_{\mathrm{s}}-x_{\mathrm{r}}$

so that the desired signal alone becomes visible when averaging. Typically, each of the two measurements are done at different times, after the telescope has moved between two positions on sky.

In order to obtain a plausible estimate of the error of the difference we use the standard definition of the variance:

$\sigma_{d}^{2}(T)=\left\langle(d-\langle d\rangle)^{2}\right\rangle=\left\langle d^{2}\right\rangle-\langle d\rangle^{2}$.

The brackets " \langle\rangle " stand for the expectation value. In comparison, this definition is similar to the original definition of the Allan variance (Allan 1966), if one considers a situation, where the expectation value of the difference is zero which is practically "normal" during radio-astronomical observations:

$\sigma_{\mathrm{A}}^{2}(T)=1 / 2\left\langle d^{2}\right\rangle \cdot$

For further treatment we use the standard definition of the variance, but leave the factor of $1 / 2$ in place for historical reasons, since it was already introduced by Allan in 1966 . Thus we use $^{1}$ :

$\underline{\sigma_{\mathrm{A}}^{2}(T)=1 / 2\left\langle(d-\langle d\rangle)^{2}\right\rangle}=1 / 2\left[\left\langle d^{2}\right\rangle-\langle d\rangle^{2}\right]$.

1 This original definition through the difference of samples may be altered by using the ratio of contiguous data instead.
Note that with this new definition we consider also the possibility that the mean of the difference may not be zero. In case there is radiometric noise only, this expression defines the noise of a single measurement $x_{\mathrm{s}}$ or $x_{\mathrm{r}}$ alone thanks to the factor of $1 / 2 .^{2}$

If we apply now Eq. (1), we get:

$\sigma_{\mathrm{A}}^{2}=\left[\sigma_{\mathrm{s}}^{2}(T)+\sigma_{\mathrm{r}}^{2}(T)\right] / 2-\left[\sigma_{\mathrm{s}}^{2}(T) \sigma_{\mathrm{r}}^{2}(T)\right]^{1 / 2} g_{\mathrm{sr}}(T)$

with

$g_{\mathrm{sr}}(T)=\frac{\left\langle\left(x_{\mathrm{s}}-\left\langle x_{\mathrm{s}}\right\rangle\right)\left(x_{\mathrm{r}}-\left\langle x_{\mathrm{r}}\right\rangle\right)\right\rangle}{\left[\left\langle\left(x_{\mathrm{s}}-\left\langle x_{\mathrm{s}}\right\rangle\right)^{2}\right\rangle\left\langle\left(x_{\mathrm{r}}-\left\langle x_{\mathrm{r}}\right\rangle\right)^{2}\right\rangle\right]^{1 / 2}}$,

$\sigma_{\mathrm{s}}^{2}(T)=\left\langle\left(x_{\mathrm{s}}-\left\langle x_{\mathrm{s}}\right\rangle\right)^{2}\right\rangle$ and $\sigma_{\mathrm{r}}^{2}(T)=\left\langle\left(x_{\mathrm{r}}-\left\langle x_{\mathrm{r}}\right\rangle\right)^{2}\right\rangle \cdot$

$g_{\mathrm{sr}}(T)$ is the normalized cross-correlation function of the two data sets $x_{\mathrm{s}}$ and $x_{\mathrm{r}}$. It should be understood that the expectation values are the means averaged over the time $t$. In other cases it might be the mean of a large number of spectrometer pixels for example. Both cases should be equivalent for the discussion here.

If we have the same statistics for both, "s" and " $\mathrm{r}$ " $\left(\sigma_{\mathrm{r}}^{2}(T)=\sigma_{\mathrm{s}}^{2}(T)=\sigma^{2}(T)\right)$, then we get finally:

$\sigma_{\mathrm{A}}^{2}(T)=\sigma^{2}(T)\left[1-g_{\mathrm{sr}}(T)\right]$.

According to this expression the Allan variance is always smaller than the normal variance of the data sets as long as there is no "anti-correlation" with negative $g_{\mathrm{sr}}(T)$. The measurement of differences therefore removes all contributions from the noise which are correlated. This reflects the simple fact that the impact of slow drift noise on the signal to noise ratio can be removed by signal modulation techniques, as is commonly applied during observations in radio-astronomy or when using Lock-In amplifiers in laboratory experiments. It also tells immediately that fast switching does not help whatsoever, if there is no correlation as is typical for pure white noise.

We have not yet made any particular assumption about the source of the signal- and the reference-data. For our application here, the two data "s" and "r" are derived from the same output signal $s(t)$ of one spectrometer channel. The two acquisition periods of length $T$ for the integration of $x_{\mathrm{s}}$ and $x_{\mathrm{r}}$ must therefore occur one after the other in order to avoid any undesirable overlap between the two measurements. For an unequivocal definition of the instrumental Allan variance we assume that all "s" and "r" measurements are contiguous without any dead

The corresponding "ratio-variance" is then: $\sigma_{\mathrm{r}}^{2}(T)=1 / 2 \times$ $\left\langle\left[x_{\mathrm{s}} / x_{\mathrm{r}}-\left\langle x_{\mathrm{s}} / x_{\mathrm{r}}\right\rangle\right]^{2}\right\rangle$.

In case the rms of the noise is small as compared with the mean $\langle s(t)\rangle$, one can easily show that $\sigma_{\mathrm{r}}^{2}(T)=\sigma_{\mathrm{A}}^{2}(T) /\langle s(t)\rangle^{2}$. This new definition has the advantage to properly calibrate the data even at varying gain in the system.

${ }^{2}$ In general one has to consider the fact that there is only a finite data set available for the calculation of a variance. Therefore, instead of Eq. (3), one should use the standard definition with $\sigma_{\mathrm{A}}^{2}(T)=\frac{1 / 2}{N-1} \sum_{n=1}^{N}\left(d_{n}-\bar{d}\right)^{2}$ with $\bar{d}=$ $1 / N \sum_{n=1}^{N} d_{n}$. 
time in between. In real life, when observing, there will be always some unavoidable dead time, since the telescope needs to be moved between the On- and the Off-position or there is time needed for data transfer etc. Any delay will increase the impact of slow drift noise, and it will therefore result in a different appearance of the system noise. Such effects will be discussed in the next chapter.

\section{The role of the minimum}

For a given integration time the signal output of one spectrometer channel is described by Eq. (1). We can describe the instantaneous noise signal $s(t)$ before integrating using the (in this case not normalized) auto-correlation function $\gamma(\tau)$, but here as a function of delay time $\tau$ :

$\gamma(\tau)=\langle(s(t+\tau)-\langle s(t+\tau)\rangle)(s(t)-\langle s(t)\rangle)\rangle \cdot$

The integrated signals " $x$ " have a new auto-correlation function $\Gamma_{T}(\tau)$ :

$\Gamma_{T}(\tau)=\langle(x(T, t+\tau)-\langle x(T, t+\tau)\rangle)(x(T, t)-\langle x(T, t)\rangle)\rangle$,

and we get after some manipulation, when using Eqs. (1) and (5):

$\Gamma_{T}(\tau)=1 / T^{2} \int_{-T}^{T}(T-|t|) \gamma(t+\tau) \mathrm{d} t$.

According to the definition of the Allan variance in Eq. (3) we have now:

$\sigma_{\mathrm{A}}^{2}(T)=\Gamma_{T}(0)-\Gamma_{T}(T)$.

Frequently, instead of the auto-correlation function $\gamma(\tau)$, the noise power spectrum $S(f)$ is used for the description of noise. Since the signal $s(t)$ is real valued, one can write (see e.g. also in Barnes et al. 1971; Vessot 1976):

$$
\begin{aligned}
& \gamma(\tau)=\int_{0}^{\infty} S(f) \cos (2 \pi f \tau) \mathrm{d} f \text { and } \\
& S(f)=4 \int_{0}^{\infty} \gamma(\tau) \cos (2 \pi f \tau) \mathrm{d} \tau
\end{aligned}
$$

How the correlation function behaves in low order approximation for a noise spectrum like $S(f) \propto 1 / f^{\alpha}$ is easily found using Eq. (8) for sufficiently small $\tau>0$ :

$$
\begin{aligned}
\gamma(\tau) & =g_{\mathrm{c}}-g_{\alpha} \tau^{\alpha-1} & & \text { for } 1<\alpha \leq 3 \\
& =g_{\mathrm{c}}-g_{1} \log (\tau) & & \text { for } \alpha=1 \text { (flicker noise) } \\
& =g_{\mathrm{c}}+g_{\alpha} 1 / \tau^{1-\alpha} & & \text { for } 0<\alpha<1 \\
& =g_{0} \delta(\tau) & & \text { for } \alpha=0 \text { (white noise) }
\end{aligned}
$$

The parameters $g_{\mathrm{c}}, g_{\alpha}, g_{1}, g_{0}$ describe the actual contribution to the correlation function. In all cases we have: $\gamma(-\tau)=\gamma(\tau)$. According to Eq. (5), $\gamma(0)$ is identical with the expectation value of the square of the signal, which is equivalent to the total power contained in the noise fluctuations, and it has to be finite. Consequently, $1 / f^{\alpha}$ power spectra also have to stay finite at frequencies close to zero, at least for $\alpha \geq 1$, since the integral over the noise power spectrum $S(f)$ for zero $\tau$ must not diverge for the same reason (see Eq. (8) for $\tau \rightarrow 0$ ). It means that $1 / f^{\alpha}$ spectra cannot exist at very small $f$ ! It is easy to deal with the divergence problem by introducing a lower cut-off frequency for spectra where $\alpha \geq 1$ (see e.g. Barnes et al. 1971). On the other hand, for $0 \leq \alpha<1$ the power spectra must have an upper cut-off frequency because of the same arguments. Thus, white noise in this sense has to be "band-limited" which is automatically the case in any real experiment due to inevitable time constants for example. The special case of "flicker noise" $(\alpha=1)$ requires both, a lower and an upper cut-off frequency, in order to be realistic. Consequently, the formulas (9) are valid within limits for $\tau$, which are also defined by the appropriate cut-off frequencies. Important for the following treatment is that for $1<\alpha \leq 3 \mathrm{Eq}$. (9) is valid also for $\tau \rightarrow 0$. The range $0<\alpha \leq 1$ we do not consider any further, since these noise power spectra don't seem to be observable under normal circumstances, at least with standard radio-astronomical equipment.

In this approximation we have now for the Allan variance according to Eqs. (6), (7), and (9):

$$
\begin{aligned}
\sigma_{\mathrm{A}}^{2}(T) & =g_{\alpha} \frac{4\left(2^{\alpha-1}-1\right)}{\alpha(\alpha+1)} T^{\alpha-1} & & 1<\alpha \leq 3 \\
& =g_{0} / T & & \alpha=0 \text { (white noise). }
\end{aligned}
$$

For $\alpha>1$ Eq. (10) is valid for integration times $T$ smaller than the characteristic correlation time of the drift noise and larger than is determined by the highest frequency components of the noise. These two assumptions apply in all cases considered here.

If we assume a simple power law for the drift contribution with a well defined $\alpha$, and if we consider the additional presence of radiometric noise, or "white noise", we expect the Allan variance to have the following structure as a function of integration time:

$\sigma_{\mathrm{A}}^{2}(T)=a / T+b T^{\beta} \quad(\beta=\alpha-1)$.

It is general experience with radio-astronomical as well as ordinary laboratory equipment that the slope of the drift contribution is found somewhere between $\beta=1$ and $\beta=2$, which corresponds to $1 / f^{2}$ - and $1 / f^{3}$-noise respectively. Good examples of such correlation functions are the spontaneous decay of excited molecular states with a simple exponential correlation function, or emission from a thermal source with a Gaussian correlation function. When expanded in lowest order approximation, they result in terms with $\beta=1$ and 2 respectively. Chaotic processes will typically lead to power-laws somewhere in between. We have never found an indication of the presence of $1 / f$-noise in any of our instruments which would contribute with a horizontal slope in the Allan variance plot.

Within the white noise part of the Allan plot, i.e. the regime with the slope of " -1 ", the radiometer equation must be valid:

$\sigma_{\mathrm{A}}^{2}(T)=\frac{\langle s(t)\rangle}{B_{\mathrm{F} l} T}$ 

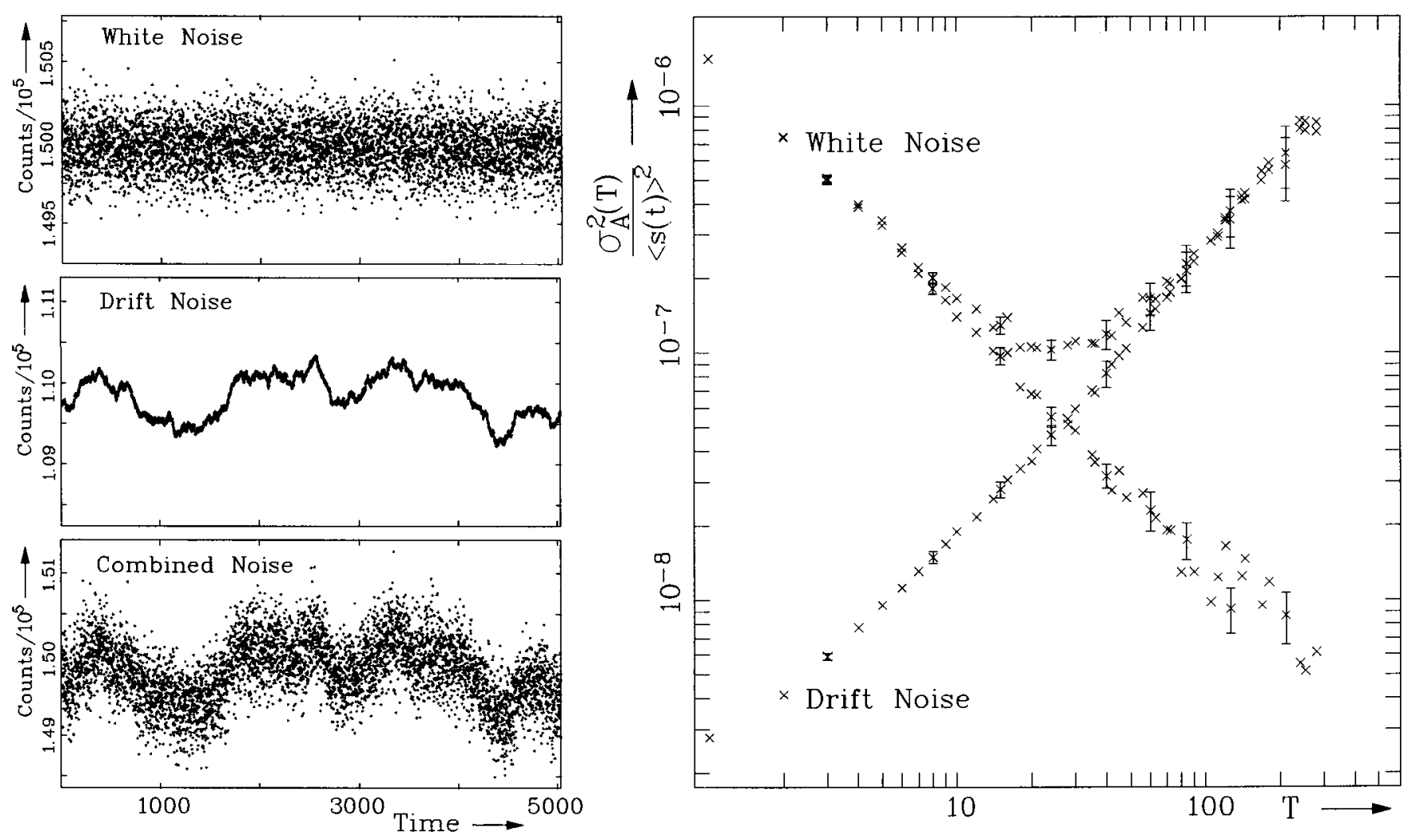

Fig. 1. Artificial data set generated by random numbers (left) with white noise of Gaussian distribution (top), drift noise (middle), and combined noise (bottom). Each data point corresponds to a sample integrated for $1 \mathrm{~s}$ while the fluctuation bandwidth was set to $600 \mathrm{kHz}$. The drift noise is calculated by filtering white noise with a sufficiently broad boxcar time-filter (width $>T_{\max }$ in the Allan variance plot). To the right the (relative) Allan variance plots of all three noise spectra are depicted. The white noise appears with a slope of -1 , the drift noise with a slope of approximately +1 . The combination of both results in a typical Allan plot with a minimum at some fairly well defined minimum time.

$B_{\mathrm{Fl}}$ is the "fluctuation bandwidth" of the spectrometer of the frequency channel of the spectrometer, which is defined as:

$B_{\mathrm{Fl}}=\frac{\left[\int_{0}^{\infty} P(f) \mathrm{d} f\right]^{2}}{\int_{0}^{\infty} P^{2}(f) \mathrm{d} f}$

(see e.g. Kraus 1980 and references therein). $P(f)$ is the power response function of the frequency channel to a monochromatic input at frequency $f . B_{\mathrm{Fl}}$ is always larger than the resolution-bandwidth $\delta_{\text {Res }}$ of the channel, so that the radiometric noise should be somewhat smaller than often is expected. Typically $B_{\mathrm{Fl}}$ is more than $50 \%$ larger than $\delta_{\text {Res }}$.

In most practical cases it is very useful to refer to the particular integration time in the Allan variance plot where the minimum occurs. This minimum describes the turn-over point where the radiometric noise with a slope of -1 in the logarithmic plot becomes dominated by the additional and undesired drift noise (see Fig. 1). Above the minimum time the rms of the measurements becomes much larger than is anticipated by the radiometer equation alone. Intuitively, the minimum time might appear as an upper limit for the integration on individual positions during radio-astronomical observations, but the Allan variance plot offers a lot more detailed advice when planning the most efficient observing strategy under the given circumstances. Since any additional noise above the radiometric level is very unfavorable, one has to find the optimum integration time, where the loss due to inevitable dead time during slew of the telescope etc. is as little as possible, and where the impact of drift contributions is nearly negligible at the same time. To find this best compromise is the goal of the following chapters.

By use of the minimum time $T_{\mathrm{A}}$ of the variance we can now rewrite the above equation with:

$$
\frac{\sigma_{\mathrm{A}}^{2}(T)}{\langle s(t)\rangle^{2}}=\frac{1}{B_{\mathrm{F} 1} T_{\mathrm{A}}}\left(1 / t+t^{\beta} / \beta\right) \text { with } t=T / T_{\mathrm{A}} .
$$

In a mathematical sense the minimum time appears rather naturally as the decisive parameter for the description of the plot. It is obvious that at the minimum the variance is already significantly larger than the radiometric value, for $\beta=1$ it is doubled for example.

The slope of the drift part in the Allan variance plot is, as is seen in Fig. 1, also one of the important parameters for the characterization of the instrument. Therefore, we can conclude that the minimum time, the fluctuation bandwidth, and the slope at large integration time are the three parameters which fully characterize the instrument in a statistical sense. All three parameters are 
directly accessible from the Allan variance plot once there are sufficient data collected for a reliable evaluation. It is interesting to note that generally the outcome of an Allan variance test looks nearly identical to previous ones as long as the instrumentation used for the test is not altered. This is particularly useful for checking the health of an instrument from time to time. Certainly, there are other methods to describe the noise performance of a radiometer like the plot of the noise power spectrum or the correlation function or else, but it seems rather natural to use the Allan variance plot, since it is directly related to the normal observing procedure when observing an "On"and an "Off-position" with a radio-telescope.

If the fluctuation bandwidth $B_{\mathrm{Fl}}$ is changed the minimum also shifts due to the changing level of white noise, but, despite the change of the leading factor, Eq. (13) is not altered due to the normalization of the time with the Allan variance minimum time. How the radiometric contribution is decreasing with increasing fluctuation bandwidth is clear from the radiometer equation. However, the drift contribution should not change, since it does not depend on the shape of the filter-function of the actual spectrometer channel. The minimum therefore shifts to smaller times with increasing $B_{\mathrm{Fl}}$ like

$T_{\mathrm{A}}^{\prime}=T_{\mathrm{A}}\left(B_{\mathrm{Fl}} / B_{\mathrm{Fl}}^{\prime}\right)^{1 /(\beta+1)}$.

This formula should help when considering the stability of the spectrometer output while co-adding adjacent pixels for example. (The problem, how the fluctuation bandwidth changes when co-adding, is not so easily solved. This is discussed in the Appendix.)

Co-adding frequency pixels of a spectrometer output is standard practice in radio-astronomy when dealing with very broad emission lines e.g. from other galaxies. Thus it is not uncommon to finally discuss spectra with an effective fluctuation bandwidth of the order of $50 \mathrm{MHz}$ by binning several spectrometer channels. A typical minimum time of a complete radiometer system at an observatory is somewhere around $30 \mathrm{~s}$ or so at a resolution of $1 \mathrm{MHz}$ of the spectrometer. According to Eq. (14) one would expect a shift of the minimum time to values somewhere between 4 and $8 \mathrm{~s}$ for the bins. A much larger bandwidth one has to deal with, when measuring continuum signals with large bandwidth bolometers. A typical effective bandwidth may be of the order of some $50 \mathrm{GHz}$. In this case the minimum of the Allan variance moves to values between 0.1 and $0.8 \mathrm{~s}$, when assuming the origin of the white noise is still just radiometric while the drift noise remains as before. It is clear that the integration time used for sampling on each position may be a few seconds in the first case, but has to be less than $100 \mathrm{msec}$ in the second.

\section{Using the information contained in the Allan variance plot}

As was mentioned above, the Allan variance plot provides information about what to expect in case there are no gaps in time between the corresponding measurements "signal" (On) and "reference" (Off). This is very close to the standard situation during observing, but now the presence of dead time has to be included into the discussion. When investigating the simple description of the Allan variance as a function of integration time from above it seems plausible that the plot should also provide all information about the impact of drift noise, if there is dead time between the two measurements. How to do this is fairly straightforward, and, in order to keep things short, we present the mathematical treatment only briefly.

\subsection{Position-Switch observations}

Position-Switch measurements with one signal integration (On) per reference measurement (Off) are very common for the observation of single positions in an extended source for example. In other cases Beam-Switch with a wobbling secondary mirror or Frequency-Switch measurements are applied, since these methods seem to be more promising for the resulting signal to noise ratio. In terms of a more mathematical treatment, all these methods are identical, only the typical time scale is different. In practice some dead time needs to be included in the observing procedure, but both, On- and Off-integration, are assumed to be of equal length ${ }^{3}$. Following Eq. (1) we have for the signal- and the reference-measurement:

$x_{\mathrm{s}}(T, t)=1 / T \int_{t-T}^{t} \mathrm{~d} t^{\prime} s\left(t^{\prime}\right)$,
$x_{\mathrm{r}}(T, t)=1 / T \int_{t+T_{\mathrm{d}}}^{t+T_{\mathrm{d}}+T} \mathrm{~d} t^{\prime} s\left(t^{\prime}\right)$

when including the delay time $T_{\mathrm{d}}$ between the end of the On-integration and the begin of the Off-integration. For the error estimate of difference of these two measurements we get now with the help of Eqs. (5), (6), and with $\sigma_{1}^{2}\left(T, T_{\mathrm{d}}\right)=\Gamma_{T}(0)-\Gamma_{T}\left(T_{\mathrm{d}}+T\right)($ similar as in Eq. $(7))$ :

$\frac{\sigma_{1}^{2}\left(T, T_{\mathrm{d}}\right)}{\langle s(t)\rangle^{2}}=\frac{2}{B_{\mathrm{Fl}} T_{\mathrm{A}}}(1 / t+1 / \beta f(t, d))$,

$t=T / T_{\mathrm{A}}, d=T_{\mathrm{d}} / T_{\mathrm{A}}$ with

$f(t, d)=t+3 / 2 d, \beta=1$, and

$f(t, d)=[t+d]^{2}, \beta=2$.

It is possible to derive suitable expressions for arbitrary values of $\beta$, but in the following treatment we concentrate on the two extreme cases $\beta=1$ and 2 only. $\sigma_{1}^{2}\left(T, T_{\mathrm{d}}\right)$ describes now the noise found with one single pair On and Off. It is most efficient to move the telescope only every second time so that the observing sequence is On-Off/OffOn/On-Off... instead of On-Off/On-Off/On-Off...

\footnotetext{
3 The assumption of equal length is only valid for identical noise levels of both measurements $x_{\mathrm{s}}$ and $x_{\mathrm{r}}$. If the emission from the two positions is very different and not small in comparison to the receiver noise temperature, an equal length of the two integrations is no longer a proper choice. This would apply when studying emission from the sun for example, but in radio-astronomy, it would be an exceptional situation.
} 
(This is also true for Beam-Switch measurements!) In this case we have for the duration of each complete cycle with one On- and one Off-integration:

$T_{\mathrm{c}}=2 T+T_{\mathrm{d}}$

Usually, the measurement is repeated several times and the result is co-added to improve the signal to noise ratio. Then we have $K$ such pairs, which are measured within a total observing time $T_{\text {Obs }}$. We get therefore for a given observing time $T_{\text {Obs }}$ :

$T_{\mathrm{Obs}}=K T_{\mathrm{c}}$.

Since the variance should develop like $1 / K$, we have finally for the variance of the complete observation on one Onposition $^{4}$.

$$
\begin{aligned}
\frac{\sigma_{\mathrm{K}}^{2}\left(T, T_{\mathrm{d}}\right)}{\langle s(t)\rangle^{2}} & =1 / K \frac{\sigma_{1}^{2}\left(T, T_{\mathrm{d}}\right)}{\langle s(t)\rangle^{2}} \\
& =\frac{4}{B_{\mathrm{Fl}} T_{\mathrm{Obs}}}(1 / t+f(t, d) / \beta)(t+d / 2) .
\end{aligned}
$$

Any realistic drift scenario can be described by this formula, and the result must be located within the range of the two limiting values of $\beta$. For a useful calculation it is now mandatory that the information about the minimum time $T_{\mathrm{A}}$ is known from an Allan variance measurement.

Figure 2 shows the shape of Eq. (16) as a function of the relative integration time $t$ for a few values of $d$. For each $d>0$ the function has exactly one fairly broad minimum, and it is plausible that only in this minimum the observation can be done with maximum efficiency. Any other $t$ leads to a higher noise level, i.e. to lower efficiency within a given observing time. This can be explained by the facts that with very short integration a lot of time is wasted while moving the telescope, and that at very long integration time the drift noise starts to deteriorate the signal to noise ratio on the other hand. In Fig. 3 the optimum integration time at the minimum of the variance is shown for both cases $\beta=1$ and 2 as a function of the relative dead time $d$. The preferred relative integration time $t$ is always significantly smaller than unity, which leads to the important conclusion that the integration time should always be considerably smaller than the Allan variance minimum time. With a realistic drift noise contribution $(1 \leq \beta \leq 2)$ the optimum integration time will be located somewhere between the two solid lines in the plot. For the figure, also those limits for the integration time have been computed, where the rms-noise is increased by less than $1 \%$ as compared to the optimum. The dotted curves indicate these limits for both $\beta$, and it is appears that these regions overlap largely. The hatched area in the plot indicates where this overlap-region is found. It means that for any realistic scenario it is always possible to find an integration time with almost perfect noise performance

${ }_{4}$ At long total observing time the reduction of the variance like $1 / K$ can be proven for any realistic noise power spectrum when using the fact that the noise correlation function must stay finite for $\tau \rightarrow 0$ (see also above). independent on the actual drift characteristics of the system. Consequently, the precise knowledge of the drift slope $\beta$ is not really essential for the optimization procedure.

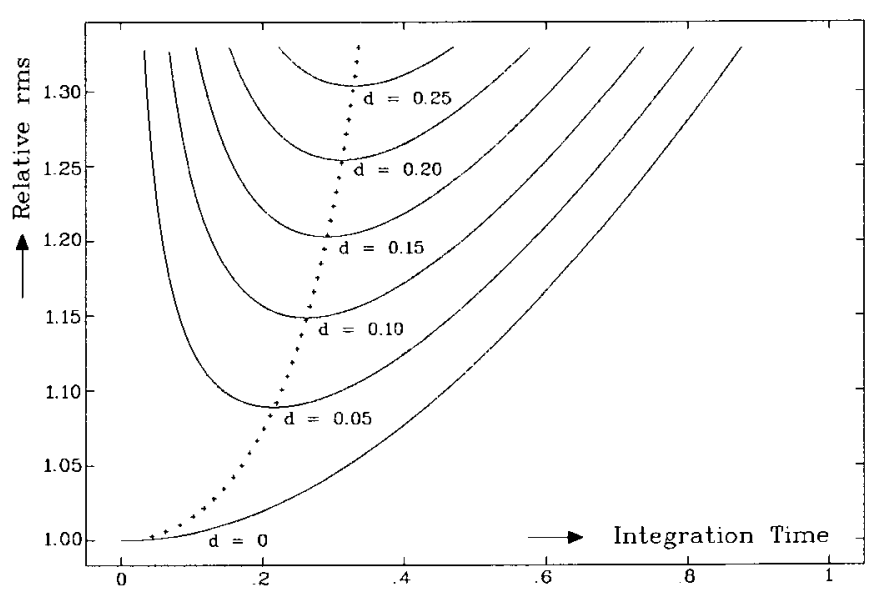

Fig. 2. The development of the rms of Position-Switch measurements as a function of integration time for a drift slope of $\beta=1$ in the Allan variance plot (see Eq. (16)). The curves are calculated for several delay times between On- and Offposition $(d=0, \ldots, 0.25)$. The dotted curve connects all minima of the curves and represents the optimum integration time for all delays. The values of the delay time $d$ as well as of the integration time are given in units of the Allan variance minimum time.

As was mentioned before, with a standard low resolution spectrometer one typically finds an Allan variance minimum of a complete radiometer system in the range of $30 \mathrm{~s}$ or so. Chopped measurements, using a wobbling secondary telescope mirror for example, are considered as the ideal method for point-like sources to reduce the impact of drift noise on the appearance of the baselines of the spectra. If the chop delay, i.e. the time to move the subreflector between the two positions, needs $100 \mathrm{msec}$ for example, the optimum integration time per position is found near $4 \mathrm{~s}$ following Eq. (16). The situation seems to be different for the case $d=0$, as it would apply for Frequency-Switch measurements for example, since the switch between the two nearby frequencies takes negligible time. But, as is visible in Fig. 2, the increase in rms noise is fairly marginal $(\leq 1 \%)$ even for integration times $T$ up to $14 \%$ of $T_{\mathrm{A}}$. This means, in all practical cases it is of no use to switch at high speed, on the contrary, the efficiency of the observation might become affected, if dead time is involved. Even for spectra at moderately reduced frequency resolution the required integration time does not drop significantly below $1 \mathrm{~s}$. It is therefore important to note, that a higher chop frequency is only required for continuum measurements with very large bandwidth.

The ideal, theoretical limit for the observing efficiency is reached, when effectively all integration time is spent 


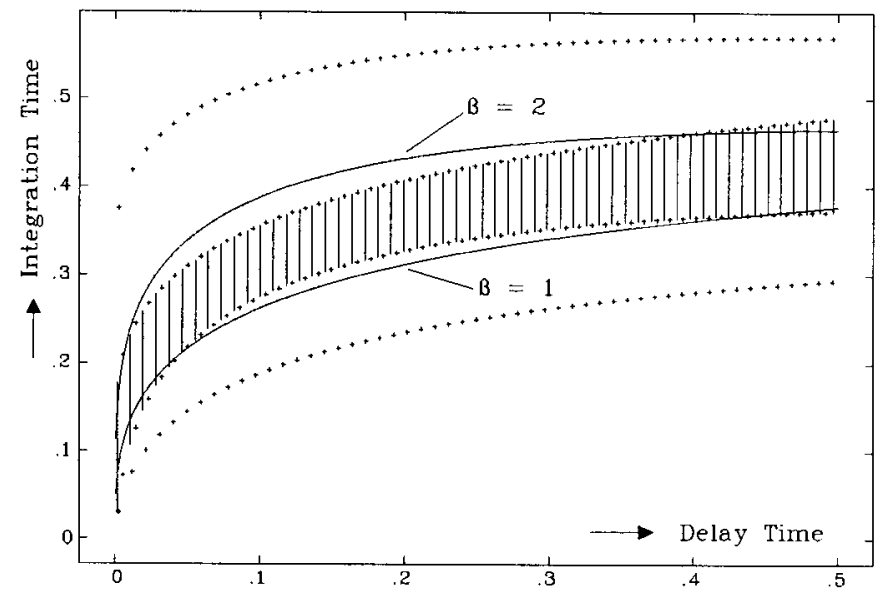

Fig. 3. Optimum integration time as a function of On-Off delay for the two extreme drift contributions with $\beta=1$ and $\beta=2$ as found from Eq. (16). The dotted curves represent the intervals where the rms is increased by $1 \%$ maximum for both values of $\beta$. The hatched area defines the regime where the rms increase is less than $1 \%$ independent on the actual value of $\beta$. In this area the preferred choice of the integration time is found. The values of the delay time $d$ as well as of the integration time are given in units of the Allan variance minimum time.

on the On-position and if there would be no dead time involved. In this case we have:

$$
\frac{\sigma_{\mathrm{th}}^{2}(T)}{\langle s(t)\rangle^{2}}=\frac{1}{B_{\mathrm{Fl}} T_{\mathrm{Obs}}} \text {. }
$$

The best possible efficiency relative to this theoretical performance is therefore:

$$
\begin{aligned}
\eta & =\left[\sigma_{\mathrm{th}}^{2}(T) / \sigma_{K}^{2}\left(T, T_{\mathrm{d}}\right)\right]^{1 / 2} \\
& =1 / 2\left[\left(1 / t_{0}+1 / \beta f\left(t_{0}, d\right)\right)\left(t_{0}+d / 2\right)\right]^{-1 / 2}
\end{aligned}
$$

with $t_{0}=T_{0} / T_{\mathrm{A}}$ the optimum integration time for the given delay. This observing efficiency $\eta$ is always smaller than $50 \%$, since at least half of the time is "wasted" for the integration of the Off-signal. Clearly, the longer the dead time the less efficient the observation. Since the impact of the dead time is determined by its relative length when comparing with the Allan variance minimum time, a larger $T_{\mathrm{A}}$ helps as well. (A plot of Eq. (17) can be found in Fig. 5.) It should be kept in mind that the efficiency calculated here is the best possible for a given $d$. If other integration times are chosen, the efficiency will definitely become worse! One should also be aware of the fact that the total observing time has to be increased by a factor proportional to the square of the inverse efficiency to compensate for the reduced efficiency, which might become a high price to pay for a non-appropriate observing strategy.

\subsection{Mapping}

Another and possibly more interesting case is the situation when measuring maps either by Raster-Mapping or On-The-Fly. In both cases there are $N$ different Onpositions per Off-position in one cycle, the only difference is that for Raster-Mapping there is some dead time between the different On-positions which does not appear during OTF observations. It is found in literature that the Off-integration time should be $\sqrt{N}$ times longer than the On-integration time (Ball 1976). This advice leaves the question open how long the On-integration should last. For the following treatment of this question we assume that we have an On-integration time $T_{\mathrm{s}}$, an Offintegration time $T_{\mathrm{r}}$, a dead time $T_{\mathrm{ds}}$ between each of the On-measurements, another dead time $T_{\mathrm{dr}}$ to move from the last On- to the Off-position, and a different dead time $T_{\mathrm{dc}}$ to move the telescope back to the first Onposition to begin with the next cycle again. It is plausible that $T_{\mathrm{dc}}$ will not be identical with $T_{\mathrm{dr}}$, since the first and last On-position are not the same, and the time to move between the positions (with different velocity requirements in OTF-mode as well) is definitely different.

The delay between one of the On-positions and the Off-position is also dependent on the number of Ons in between. If we consider the worst case situation, we have to investigate the On-Off pairs with maximum delay involved, which is the first On-position when putting the Off at the end of the cycle. The delay $T_{\mathrm{d}}$ is then:

$$
\begin{aligned}
T_{\mathrm{d}} & =(N-1)\left(T_{\mathrm{s}}+T_{\mathrm{ds}}\right)+T_{\mathrm{dr}} \text { or } \\
d & =(N-1)\left(s+d_{\mathrm{s}}\right)+d_{\mathrm{r}} .
\end{aligned}
$$

Here and for the following we use $d=T_{\mathrm{d}} / T_{\mathrm{A}}, d_{\mathrm{s}}=T_{\mathrm{ds}} / T_{\mathrm{A}}$, $d_{\mathrm{r}}=T_{\mathrm{dr}} / T_{\mathrm{A}}, d_{\mathrm{c}}=T_{\mathrm{dc}} / T_{\mathrm{A}}, s=T_{\mathrm{s}} / T_{\mathrm{A}}$ and $r=T_{\mathrm{r}} / T_{\mathrm{A}}$.

We also have to take into account now that the integration time for On is different than for Off. Hence we write:

$$
\begin{aligned}
& x_{\mathrm{s}}\left(T_{\mathrm{s}}, t\right)=1 / T_{\mathrm{s}} \int_{t-T_{\mathrm{s}}}^{t} s\left(t^{\prime}\right) \mathrm{d} t^{\prime} \\
& x_{\mathrm{r}}\left(T_{\mathrm{r}}, t\right)=1 / T_{\mathrm{r}} \int_{t+T_{\mathrm{d}}}^{t+T_{\mathrm{d}}+T_{\mathrm{r}}} s\left(t^{\prime}\right) \mathrm{d} t^{\prime} .
\end{aligned}
$$

Similar as before we find after some straight-forward derivation using Eqs. (5), (6), (9), and (19):

$$
\sigma_{1}^{2}(s, r)=\Gamma_{T_{\mathrm{r}}}(0)+\Gamma_{T_{\mathrm{s}}}(0)-\left[w_{+} \Gamma_{T_{+}}\left(T_{m}\right)-w_{-} \Gamma_{T_{-}}\left(T_{m}\right)\right]
$$

with $T_{ \pm}=\left(T_{\mathrm{r}} \pm T_{\mathrm{s}}\right) / 2, w_{ \pm}=2 T_{ \pm}^{2} /\left[T_{\mathrm{r}} T_{\mathrm{s}}\right]$, and $T_{\mathrm{m}}=T_{+}+$ $T_{\mathrm{d}}$. When integrating one finds now:

$\frac{\sigma_{1}^{2}(s, r)}{\langle s(t)\rangle^{2}}=\frac{1}{B_{\mathrm{Fl}} T_{\mathrm{A}}}(1 / s+1 / r+2 g(s, r, d) / \beta)$

and for the two limiting cases of $\beta$ one gets: $g(s, r, d)=(s+r) / 2+3 / 2 d, \beta=1$, and $g(s, r, d)=[(s+r) / 2+d]^{2}, \beta=2$.

The function $g(s, r, d)$ is identical with $f(t, d)$ for $s=$ $r=t$ (see Eq. (15)). The variance found here is valid for one pair of a particular On- and the corresponding Off-measurement.

We have to identify now, how the noise is developing, if one wants to observe a full map within a given 
total observing time $T_{\text {Obs }}$. One observing cycle consists of $N$ identical On-integrations $\left(T_{\mathrm{s}}\right)$, one Off-integration $\left(T_{\mathrm{r}}\right)$, and the various dead times in between. Thus we have for the complete cycle time $T_{\mathrm{c}}$ :

$T_{\mathrm{c}}=N T_{\mathrm{s}}+T_{\mathrm{r}}+(N-1) T_{\mathrm{ds}}+T_{\mathrm{dr}}+T_{\mathrm{dc}}$.

We assume that we want to measure a map consisting of $L$ different On-positions. This needs $L / N$ cycles for observing each position once. Each of the On-positions may be measured $K$ times within the total observing time $T_{\mathrm{Obs}}$ in order to improve the noise level. Thus we have:

$T_{\mathrm{Obs}}=K T_{\mathrm{c}} \times L / N$

with $K \geq 1$. The choice of $K$ may be dependent on $N, L$ and the available total observing time $T_{\mathrm{Obs}}$, and it has to be chosen according to the individual needs of the observing program. In many cases $K$ will be equal to 1 . When using Eqs. (20), (21), and (22), we get now finally:

$$
\begin{aligned}
\frac{\sigma_{K}^{2}(s, r, N)}{\langle s(t)\rangle^{2}}=1 / K \frac{\sigma_{1}^{2}(s, r, N)}{\langle s(t)\rangle^{2}} & \\
=L \frac{1}{B_{\mathrm{Fl}} T_{\mathrm{Obs}}} & (1 / s+1 / r+2 / \beta g(s, r, d)) \\
& \times\left(s+d_{\mathrm{s}}+\frac{r+d_{\mathrm{r}}+d_{\mathrm{c}}-d_{\mathrm{s}}}{N}\right) .
\end{aligned}
$$

We have found now the variance as a function of three variables $s, r$, and $N$ with the relative delays $d_{\mathrm{s}}, d_{\mathrm{r}}$, and $d_{\mathrm{c}}$ as parameters. Note that the On-Off delay $T_{\mathrm{dr}}$ has different impact on the statistics than the return delay $T_{\mathrm{dc}}$, since the latter does not affect the drift contribution $g(s, r, d)$ (see Eqs. (18), (20), (21), and (23)).

The minimum of $\sigma_{K}^{2}(s, r, N)$ can be found, where all derivatives with respect to $s, r$, and $N$ become zero. This is the set of variables where the observing efficiency becomes the best possible under the given circumstances. (It is simple to prove that there is exactly one minimum as long as $s, r$ and $N$ are larger than zero.) Any other set of variables will result in a degradation of the observing efficiency. But, as was mentioned before, the use of the relation $r=s \sqrt{N}$ leads to results very close to this optimum $^{5}$. Therefore, for all practical purposes it is sufficient to apply only a two-dimensional optimization for the two variables $s$ and $N$ :

$$
\begin{aligned}
\partial \sigma_{K}^{2}(s, r, N) /\left.\partial s\right|_{r=s \sqrt{N}} & =0 \text { and } \\
\partial \sigma_{K}^{2}(s, r, N) /\left.\partial N\right|_{r=s \sqrt{N}} & =0 .
\end{aligned}
$$

It is trivial to show that the optimum number of Ons becomes infinite in case of OTF measurements $\left(d_{\mathrm{s}}=0\right)$.

\footnotetext{
${ }^{5}$ Using Eq. (23) it is easy to verify this relation when assuming that there is no drift contribution involved. But, if there is drift noise, it is also clear from Eq. (23) that the relation is no longer valid. However, a comparison of the results of a calculation with and without the relation between On- and Off-time shows that the minimum rms-values differ only by amounts of the order of $0.1 \%$ or less. Therefore the introduction of the simple relation between $s$ and $r$ remains justified.
}

Therefore it seems to be advisable to use fairly large $N$ in order to be as close as possible to the optimum case of $N \rightarrow \infty$. On the other hand, the optimum integration time $t_{\mathrm{s}}$ becomes extremely small in this case (see below), which finds it's limitation because of hardware constraints for example. Surprisingly, for Raster-Mapping with $d_{\mathrm{s}} \neq 0$ there is always a finite $N$ required for an optimized observation. This optimum $N$ is dependent on $d_{\mathrm{s}}, d_{\mathrm{r}}$, and $d_{\mathrm{c}}$.

Usually, it is rather difficult to make observations with an arbitrary number of Ons per Off at a given geometry of a particular map. It is therefore much more interesting to derive conclusive estimates for an optimized observation under the assumption of a predefined and fixed $N$ for both, Raster-Mapping and OTF observations. In this case one has to find the minimum with:

$\partial \sigma_{K}^{2}(s, r, N) /\left.\partial s\right|_{N \text { fixed }, r=s \sqrt{N}}=0$

In any case one has to investigate what impact the chosen $N$ has on the total efficiency using Eqs. (23) and (24) in order to verify that the used $N$ is not too far away from optimum.

In order to provide some idea about the best choice of the On-observing time $s$, the optimum integration time in OTF mode is shown in Fig. 4 as a function of the On-Off delay $d_{\mathrm{r}}$. The delay for the return to the begin of the cycle is taken into account by a $d_{\mathrm{c}} 20 \%$ longer than $d_{\mathrm{r}}$. The two solid curves are derived from Eqs. (23) and (20) for the two limiting cases $\beta=1$ and $\beta=2$. The hatched area in the plot represents the region where the increase of the rms stays below $1 \%$ as compared to the optimum for both values of $\beta$. This means that for all assumed drift slopes one is always safe when choosing an On-integration time within this region. Such optimized integration time can be described by the purely empirical formula:

$s \approx 0.53 d^{0.23} / N^{0.69}, r=s \sqrt{N}$

with $d=(N-1) d_{\mathrm{s}}+d_{\mathrm{r}}+d_{\mathrm{c}}$.

$d$ is the sum of all delays in one cycle. The formula is also valid for Raster-Mapping and Position-Switch measurements, and it may be used for values of $d_{\mathrm{r}}$ and $d_{\mathrm{c}}$ between 0 and 1 , for $d_{\mathrm{s}} \leq 0.1$, and $N \geq 1$.

Finally, also the overall observing efficiency can be found for the measurement of extended maps. The theoretically best possible value of the variance is given by:

$$
\frac{\sigma_{\mathrm{th}}^{2}(s, r, N)}{\langle s(t)\rangle^{2}}=L \frac{1}{B_{\mathrm{Fl}} T_{\mathrm{Obs}}},
$$

where no dead time is present and virtually all observing time is spent on the On-positions. In this case we have now for the relative efficiency:

$$
\begin{aligned}
\eta= & {\left[\sigma_{\mathrm{th}}^{2}(s, r, N) / \sigma_{K}^{2}(s, r, N)\right]^{1 / 2} } \\
=[(1 / s+ & 1 / r+2 / \beta g(s, r, d)) \\
& \left.\quad \times\left(s+d_{\mathrm{s}}+\frac{r+d_{\mathrm{r}}+d_{\mathrm{c}}-d_{\mathrm{s}}}{N}\right)\right]^{-1 / 2}
\end{aligned}
$$

Figure 5 depicts the optimum efficiency according to Eq. (27) and (25) for three different 


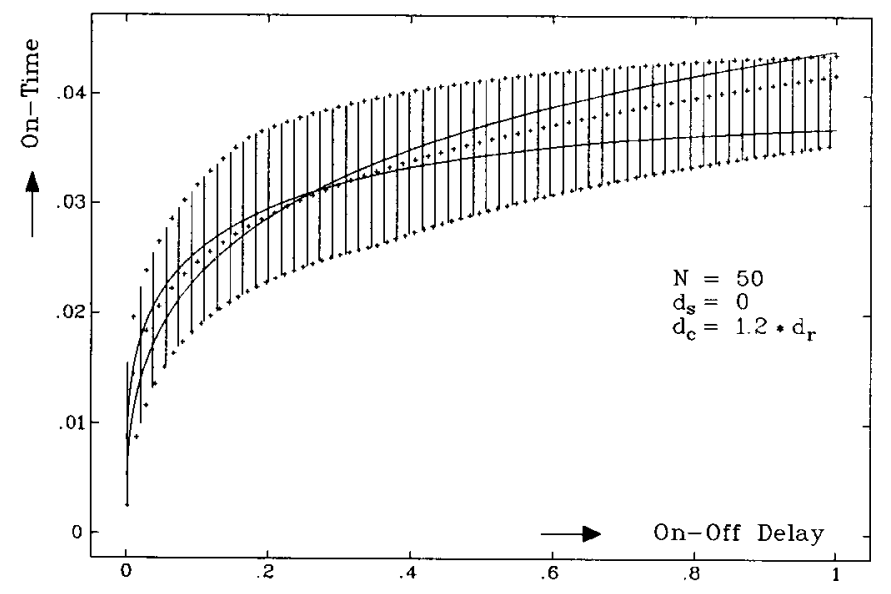

Fig. 4. Optimum On-integration time for OTF measurements with 50 Ons per Off. The hatched area represents the regime where the rms increase stays below $1 \%$ for any $\beta$ between 1 and 2 . The dotted curve in the middle represents the suggested On-integration time using Eq. (26). As is clearly visible, the optimum integration time is typically of the order of a few seconds when assuming an Allan variance minimum time near or above $100 \mathrm{~s}$.

$N(N=1,10$, and 100). The curves for $N=1$ (dotted lines) are the Position-Switch efficiencies at the same time (see Eq. (17)). Clearly the OTF efficiency is much better than the Position-Switch efficiency. At zero delay it reaches a maximum value of $(1+1 / \sqrt{N})^{-1}$, and it decreases monotonically with increasing $d_{\mathrm{r}}$. Again, the efficiency shown in the plot is the maximum one can achieve under the given circumstances. When comparing $\eta(N=10)$ with $\eta(N=100)$, it is clear that $N=100$ is the preferable choice. This example demonstrates that it is advisable to determine whether the number of desired $N$ is a reasonable choice or should be reconsidered when planning the best strategy for the observation.

How the efficiency develops with $N$ is visible in Fig. 6 for some fixed On-Off delays. Obviously, the gain in efficiency with increasing $N$ above $N=50$ is rather marginal. Therefore it is questionable whether a significant improvement in observing efficiency is achievable when going from $N=50$ to $N=100$ for example. Any reduction of the OnOff delay time would be a much more effective measure. On the other hand, the plot shows also, how valuable an increase in $N$ can be in case one is considering $N=10$ or less.

One of the remaining questions is, how long one cycle $T_{\mathrm{c}}$ will last, once the optimum On- and Off-integration time has been found. Using Eq. (21) it is now simple to calculate $T_{\mathrm{c}}$ as a function of the On-Off delay time $d_{\mathrm{r}}$. In Fig. 7 the cycle time is plotted for three cases with $N=$ 1, 10, and 100. At first sight it appears surprising that the time for a full cycle increases to values several times longer than the Allan variance minimum time in case there is substantial delay $d_{\mathrm{r}}$. But again, the length of one cycle depends strongly on the number of Ons per Off. Since the On-integration time is rather small at large $N$, the larger

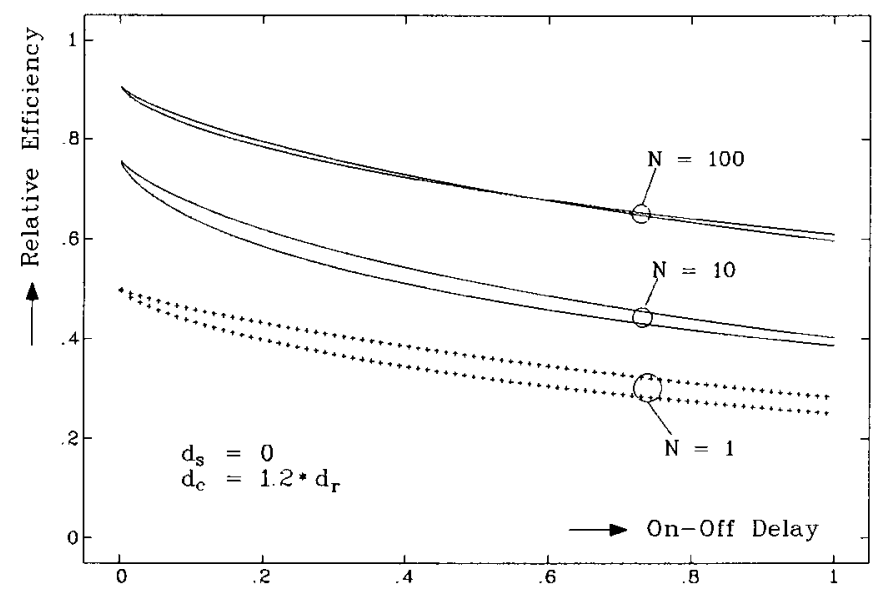

Fig. 5. Relative optimum efficiencies of OTF measurements for $N=1,10$, and 100 On-positions per Off (see Eq. (27)). For each $N$ both curves for $\beta=1$ and $=2$ are plotted. It is obvious that larger $N$ lead to higher efficiency. The dotted curves for $N=1$ represent the Position-Switch situation with an On-Off delay every second time only. This is taken into account by setting $d_{\mathrm{c}}=d_{\mathrm{s}}=0$ in Eqs. (23) and (27) while $N=1$.

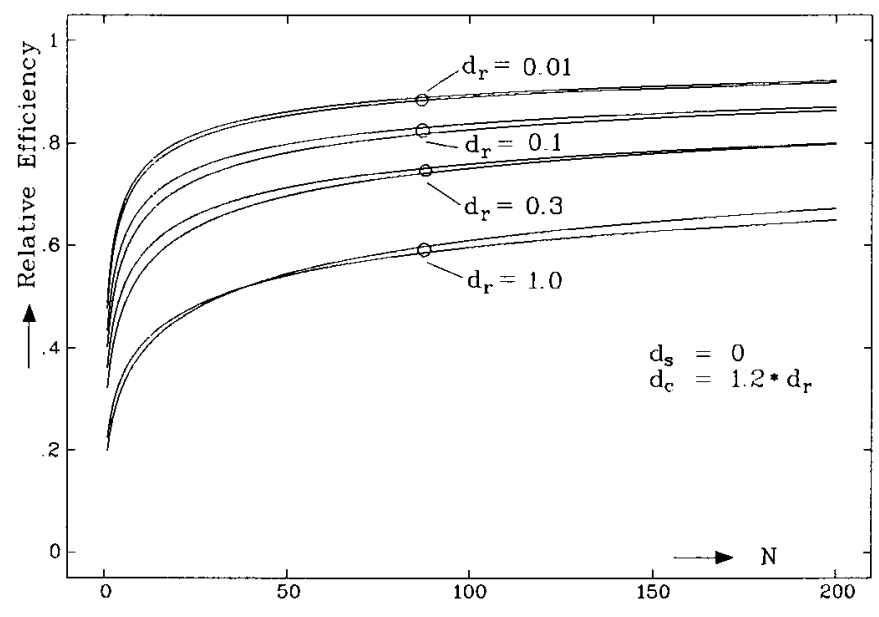

Fig. 6. Relative OTF efficiency as a function of the number of Ons per Off for various relative On-Off delays according to Eqs. (27), (25), and (23). For each $d_{\mathrm{r}}$ both curves for $\beta=1$ and $=2$ are plotted.

radiometric noise of the On-measurement dominates the noise budget so that a longer delay with an increased contribution of drift noise becomes acceptable. For a given and fixed $N$ the increase of the cycle time with increasing delay is the consequence of the fact that at larger integration time the loss due to drift noise is less costly than the loss due to the On-Off delay. This effect is also clearly visible in Fig. 2 for the case of Position-Switch measurements.

\section{Conclusion}

The discussion above provides some clear guidelines for an optimized observing program. The first step has to be a reliable measurement of the system Allan variance. The word "system" includes all components of the 


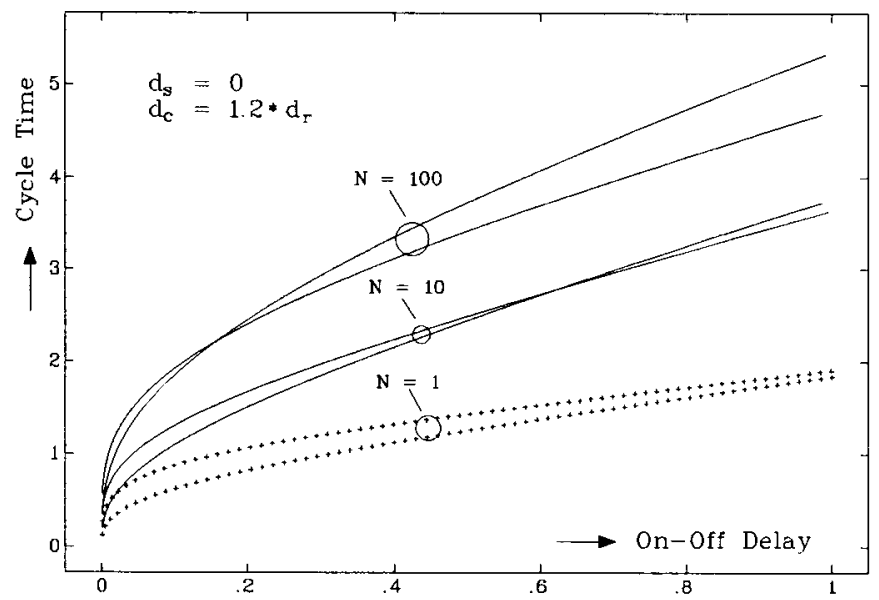

Fig. 7. Cycle time for OTF measurements as a function of On-Off delay. The cycle time comprises $N$ On-integrations, one Off-integration, and the dead times in between. The three cases $(N=1,10$, and 100) are calculated from Eqs. (21), (23), and (25). Similar to Fig. 5, the Position-Switch situation is also indicated by the dotted lines. Note that the increase of cycle time is partly due to the time spent during slew from On to Off and back.

observatory which may possibly contribute to the noise including the atmospheric fluctuations for example. When knowing the applicable dead times, a simple calculation of the optimum integration time can be made by using the "rule of the thumb" as given by Eq. (26). As was pointed out before, Position-Switch or Chop measurements should be done in a most economical way by moving the telescope or the chopper only every second time. OTF or RasterMapping measurements need a clear understanding of the impact of the number of On-positions chosen for each Offintegration. Also here it might be of some value to reverse the sequence of the integrations on the various positions every second time in order to reduce some of the loss in time due to the slew of the telescope between the On- and the Off-positions. It should be noted that the measurement of large maps can be handled in different ways. If one wants to achieve a certain signal to noise, it might be advisable to use larger $N$ with smaller $T_{\mathrm{s}}$ and to repeat the map several times, as it is considered by the parameter $K$ in Eq. (22). In any case, the suggested On- and Off-integration time should not be drastically altered, although the plot in Fig. 4 indicates that there is quite some margin available.

In general it is surprising how closely together the curves for the different $\beta$ in Figs. 5-7 are found, which is a clear validation for the assumption that it is sufficient to consider only the extreme cases for the drift contributions. Therefore, there is no need to go too deeply into the analysis of the drift part in the noise. It is also one of the better news from the treatment here that some freedom to plan the observation is still preserved. This might be particularly important when considering the constraints set by the observatory hardware. It is probably not advisable to operate with too short integration intervals, since the data flood might become overwhelming, and the storage capacity of the computers could easily be exceeded. Therefore, the conclusion found before that there are no real requirements for high speed observing most of the time is very important.

The discussion above is most useful for observations with space-born observatories like SWAS (Melnick et al. 2000), ODIN (Hjalmarson 1993) or FIRST (de Graauw et al. 1998 $)^{6}$. Since usually a satellite cannot be oriented in space very rapidly, the impact of dead time becomes vital. The SWAS satellite is not capable to control the pointing very accurately during slew across an extended source, so that the OTF mode is not applicable. Instead, Raster-Mapping is a generally used procedure. On the other hand, since SWAS is a very small satellite, it can be pointed from one position to a second in 3 degrees distance within less than 15 s. A 3-degree nod is often required during observations in the Milky Way, since the emission of molecules like CO is fairly extended. Nevertheless, the loss in observing efficiency looks acceptable, when considering an Allan variance minimum time of the SWAS receiver/backend system of about $150 \mathrm{~s}$ as found in orbit. On the Herschel space observatory, the situation will be changed drastically. We can assume that the pointing of the telescope during slew is well defined so that OTF measurements should be applicable. But, due to the fact that Herschel is going to be a very heavy satellite, the movement by three degrees will last nearly as long as the expected Allan variance minimum time will amount to. In consequence, the value of the dead times $d_{\mathrm{r}}$ and $d_{\mathrm{c}}$ will be close to unity when assuming a similar system stability like that of SWAS. This prohibits Position-Switch measurements with the instrument, because the efficiency would drop to values below $30 \%$, which would certainly be rather disappointing because of the consequences for the extremely precious and limited observing time. Therefore, a very careful analysis for determining the best possible observing strategy is extremely important for such a program.

Rather different circumstances exist at ground-based observatories. Typical dead time for a slew of 3 degrees is of the order of a few seconds only, therefore the impact of dead time does not appear as devastating as with spacebased observatories. A detailed planning of an observing strategy does not seem to be so easily implemented, particularly, if other parameters like varying hardware constraints or human limitations are playing a significant role as well. Typically, the Allan variance minimum time of most ground-based sub-millimeter observatories is rather small, partly due to the impact of an unstable atmosphere. Therefore, the advantage of a smaller dead time is partly eaten away by the reduced stability. But still, as should be clear from the discussion before, the actual situation has to be analyzed in detail for every individual case in order to achieve as much scientific return from the observations

\footnotetext{
${ }^{6}$ FIRST was recently renamed to "Herschel Space Observatory".
} 
as possible. For this the usage of the analysis presented in this paper could be very essential.

\section{Appendix A: The development of noise when co-adding frequency pixels}

Co-adding a couple of pixels in a measured spectrum in order to improve the signal to noise ratio is general practice when dealing with noisy spectra, but, the consequences of this procedure are not quite as trivial as one would like to believe. For the discussion we start again with the definition of the normalized first order correlation function as defined in Eq. (4):

$g_{m}=\left\langle\mathrm{d} y_{n} \mathrm{~d} y_{n+m}\right\rangle /\left[\left\langle\mathrm{d} y_{n}^{2}\right\rangle\left\langle\mathrm{d} y_{n+m}^{2}\right\rangle\right]^{1 / 2}$

with $\mathrm{d} y_{n}=y_{n}-\langle y\rangle$.

The data $y_{n}$ are here the pixel components of a fully calibrated spectrum as measured with a multi-channel spectrometer. The index "m" describes, by how many pixels the spectrum is shifted before the multiplication of the pixel data is done ${ }^{7}$. The correlation function is symmetric, since $g_{-m}=g_{m}$. We assume that all $y_{n}$ behave identically in a purely statistical sense. Then, the values of $g_{m}$ depend only on the "distance" between the data given by the parameter " $m$ ", and the expectation values as defined by the brackets become independent on $n$. We have to determine now the expected statistics of the new co-added data set $z_{n}$ with:

$z_{n}=1 / K \sum_{k=1}^{K} y_{n+k}$

with $K$ the number of co-added pixels. With the usual definition of the variance, $\sigma_{K}^{2}=\left\langle z_{n}^{2}\right\rangle-\left\langle z_{n}\right\rangle^{2}$, we can now determine how the error of the new data develops:

$$
\begin{aligned}
& \sigma_{K}^{2}=\left\langle\left[1 / K \sum y_{n+k}\right]^{2}\right\rangle-\left\langle 1 / K \sum y_{n+k}\right\rangle^{2} \\
& =\left\langle\left[1 / K \sum \mathrm{d} y_{n+k}\right]^{2}\right\rangle \\
& =1 / K^{2} \sum_{p=1}^{K} \sum_{q=1}^{K}\left\langle\mathrm{~d} y_{n+p} \mathrm{~d} y_{n+q}\right\rangle \\
& =\sigma_{1}^{2} / K^{2} \sum_{p=1}^{K} \sum_{q=1}^{K} g_{p-q}=\sigma_{1}^{2} / K\left[1+2 \sum_{m=1}^{K-1}(1-m / K) g_{m}\right]
\end{aligned}
$$

7 In case of a finite data set with $N$ data we can convert the definition into a more practical definition using:

$g_{m}=\frac{\frac{1}{N-m-1} \sum_{n=1}^{N-m} \delta y_{n} \delta y_{n+m}}{\left(\frac{1}{N-m-1} \sum_{n=1}^{N-m} \delta y_{n}^{2} \frac{1}{N-m-1} \sum_{n=1}^{N-m} \delta y_{n+m}^{2}\right)^{0.5}}$

with $\delta y_{n}=y_{n}-1 /(N-m) \sum_{k=1}^{N-m} y_{k}$ and $\delta y_{n+m}=y_{n+m}-$ $1 /(N-m) \sum_{k=1}^{N-m} y_{k+m}$. The expectation values are estimated here by the means over a sufficiently large number of data $(=N-m)$. Important is to note that the value of this autocorrelation function is " 1 " for $m=0$ by definition. $\sigma_{1}^{2}$ is the variance of the statistical distribution of the initial data $y_{n}$. From this and the radiometer equation we get now finally:

$\sigma_{K}^{2}=\langle z\rangle^{2} /\left[B_{K} T\right]=\sigma_{1}^{2} / K_{\mathrm{Box}}=\langle y\rangle^{2} /\left[K_{\mathrm{Box}} B_{1} T\right]$

with $K_{\text {Box }}=K /\left(1+2(1-1 / K) g_{1}+2(1-2 / K) g_{2}+\ldots\right)$. The new fluctuation bandwidth $B_{K}$ is therefore $K_{\text {Box }}$ times larger than the fluctuation bandwidth $B_{1}$ of a single spectrometer pixel. But, the effective number of pixels $K_{\text {Box }}$ is significantly smaller than the number of co-added pixels since the values of the auto-correlation function are all positive under normal circumstances. Note that the ratio of $K$ and $K_{\text {Box }}$ is a function of $K$ itself so that one has to analyze the situation for the individual case accordingly.

Only the first few values of $g_{m}$ ( $m$ not larger than about 3 ) should be non-zero for a decent spectrometer, since the overlap of the power response functions between neighbored pixels should be small. Therefore, in the limiting case of very large width of the bins ( $K$ large), we get now:

$K_{\mathrm{Box}} \approx K /\left(1+2 g_{1}+2 g_{2}+2 g_{3}\right)$.

Typical values for $K_{\text {Box }}$ at large $K$ - for instance at Nyquist sampling of the spectrum - are somewhere near $K / 2$ depending on the actual spacing and shape of the spectrometer channels, but they may vary for different spectrometer types.

\section{References}

Allan, D. 1966, Proc. IEEE, 54, 221

Ball, J. 1976, in Methods of Experimental Physics, Part C, ed. M. Meeks (Academic Press)

Barnes, J., Chi, A., Cutler, L., et al. 1971, IEEE Trans. Instrum. Meas. IM-20, 2, 105

de Graauw, T., Whyborn, N., van de Stadt, H., et al. 1998, in Proc. Advanced Technology MMW, Radio, and Terahertz Telescopes, SPIE-3357, ed. T. Philips (Kona, Hawaii: Academic Press), 336

Hjalmarson, G. 1993, in The physics and chemistry of the interstellar medium, ed. G. Winnewisser \& G. Pelz (Berlin: Springer Verlag), 369

Kooi, J., Chattopadhyay, G., Thielman, M., Phillips, T., \& R., Schieder 2000, Int. J. Infrared, \& Millim. Waves, 8, 689

Kraus, J. 1980, Radio Astronomy (Cygnus Quasar Books, 2nd edition, Chapter 7)

Melnick, G., Stauffer, J., Ashby, M., et al. 2000, ApJ, 539, 77

Rau, G., \& Schieder, R. 1984, in Proc. 14th European Microwave Conf., Liège, Belgium, 248

Schieder, R., Rau, G., \& Vowinkel, B. 1985, in Instrumentation for Submillimeter Spectroscopy, SPIE-0598, ed. E. Kollberg (Academic Press), 189

Tolls, V., Schieder, R., \& G., Winnewisser 1989, Exper. Astron., 1, 101

Vessot, R. 1976, in Methods of Experimental Physics, Part C, ed. M. Meeks (Academic Press) 